\title{
Almost $\lambda_{r}$-Statistical and Strongly Almost $\lambda_{r}$-Convergence of Order $\beta$ of Sequences of Fuzzy Numbers
}

\author{
Mahmut Işı1 ${ }^{1}$ and Mikail $\mathrm{Et}^{2,3}$ \\ ${ }^{1}$ Faculty of Education, Harran University, Osmanbey Campus, 63190 Şanliurfa, Turkey \\ ${ }^{2}$ Department of Mathematics, Firat University, 23119 Elaziğ, Turkey \\ ${ }^{3}$ Department of Mathematics, Siirt University, 56100 Siirt, Turkey \\ Correspondence should be addressed to Mikail Et; mikailet68@gmail.com
}

Received 30 September 2014; Revised 21 January 2015; Accepted 22 January 2015

Academic Editor: Ismat Beg

Copyright (C) 2015 M. Işı and M. Et. This is an open access article distributed under the Creative Commons Attribution License, which permits unrestricted use, distribution, and reproduction in any medium, provided the original work is properly cited.

\begin{abstract}
The main purpose of this article is to introduce the concepts of almost $\lambda_{r}$-statistical convergence and strongly almost $\lambda_{r}$-convergence of order $\beta$ of sequences of fuzzy numbers with respect to an Orlicz function. We give some relations between strongly almost $\lambda_{r}-$ convergence and almost $\lambda_{r}$-statistical convergence of order $\beta$ of sequences of fuzzy numbers.
\end{abstract}

\section{Introduction}

The concept of fuzzy set was introduced by Zadeh [1]. Matloka [2] introduced sequences of fuzzy numbers and provided that every convergent sequence of fuzzy numbers is bounded. Recently, sequences of fuzzy numbers have been discussed by Altınok et al. [3, 4], Aytar and Pehlivan [5], Çolak et al. [6, 7], Gökhan et al. [8], Nuray [9], and Talo and Başar [10].

The idea of statistical convergence was introduced by Fast [11] and the notion was linked with summability theory by Alotaibi et al. [12], Connor [13], Et et al. [14, 15], Fridy [16], Işık [17], Mohiuddine et al. [18, 19], and Tripathy [20]. Recently, the notion was generalized by Gadjiev and Orhan [21], Çolak [22], and Çolak and Bektaş [23].

In this paper, we study the concepts of and examine some properties of almost $\lambda_{r}$-statistical convergence and strongly almost $\lambda_{r}$-convergence of order $\beta$ of sequences of fuzzy numbers. The results which we obtained in this study are much more general than those obtained by Başarır et al. [24].

\section{Definitions and Preliminaries}

A fuzzy set $u$ on $\mathbb{R}$ is called a fuzzy number if it has the following properties:

(i) $u$ is normal; (ii) $u$ is fuzzy convex;

(iii) $u$ is upper semicontinuous;

(iv) $\operatorname{supp} u=\operatorname{cl}\{x \in \mathbb{R}: u(x)>0\}$ is compact, where cl denoted the closure of the enclosed set.

A sequence $X=\left(X_{k}\right)$ of fuzzy numbers is a function $X$ from the set $\mathbb{N}$ of all positive integers into $L(\mathbb{R})$, where $L(\mathbb{R})$ is fuzzy number space. A sequence $X=\left(X_{k}\right)$ is said to be bounded if the set $\left\{X_{k}: k \in \mathbb{N}\right\}$ is bounded. A sequence $X=$ $\left(X_{k}\right)$ is said to be convergent if there exists a positive integer $k_{0}$ such that $d\left(X_{k}, X_{0}\right)<\varepsilon$ for $k>k_{0}$, for every $\varepsilon>0$. By $w^{\mathscr{F}}$, $\ell_{\infty}^{\mathscr{F}}$, and $c^{\mathscr{F}}$ we denote the set of all, bounded, and convergent sequences of fuzzy numbers, respectively [2].

An Orlicz function is a function $N:[0, \infty) \rightarrow[0, \infty)$, which is continuous, nondecreasing, and convex with $N(0)=$ $0, N(x)>0$ for $x>0$, and $N(x) \rightarrow \infty$ as $x \rightarrow \infty$.

The generalized de la Vallée-Pousion mean is defined by $t_{m}(x)=\left(1 / \lambda_{m}\right) \sum_{k \in I_{m}} x_{k}$, where $\lambda=\left(\lambda_{m}\right)$ is a nondecreasing sequence of positive numbers such that $\lambda_{m+1} \leq \lambda_{m}+1, \lambda_{1}=1$, and $\lambda_{m} \rightarrow \infty$ as $m \rightarrow \infty$ and $I_{m}=\left[m-\lambda_{m}+1, m\right]$.

The space $\widehat{c}$ was introduced by Lorentz [25] and Maddox [26] has defined $x$ to be strongly almost convergent to a number $L$ if $\lim _{m \rightarrow \infty}(1 / m) \sum_{k=1}^{m}\left|x_{k+n}-L\right|=0$, uniformly in $n$. 
Definition 1. Let $\lambda=\left(\lambda_{m}\right)$ be as above, let $N$ be an Orlicz function, let $r=\left(r_{k}\right)$ be any sequence of strictly positive real numbers, and let $0<\beta \leq 1$ be given. A sequence $X=\left(X_{k}\right)$ is said to be almost $\lambda_{r}$-statistically convergent of order $\beta$ to $X_{0}$, with respect to the Orlicz function $N$, if for every $\varepsilon>0$

$$
\begin{array}{r}
\lim _{m \rightarrow \infty} \frac{1}{\lambda_{m}^{\beta}}\left|\left\{k \in I_{m}:\left[N\left(\frac{d\left(t_{k n}(X), X_{0}\right)}{s}\right)\right]^{r_{k}} \geq \varepsilon\right\}\right|=0, \\
\text { uniformly in } n, s>0,
\end{array}
$$

where

$$
t_{k n}(X)=\frac{X_{n}+X_{n+1}+\cdots+X_{n+k}}{k+1}=\frac{1}{k+1} \sum_{i=0}^{k} X_{n+i}
$$

The set of all almost $\lambda_{r}$-statistically convergent sequences of order $\beta$ with respect to the Orlicz function $N$ will be denoted by $\widehat{S}_{\beta}^{\mathscr{F}}(N, r, \lambda)$. In this case we write $X_{k} \rightarrow X_{0}\left(\widehat{S}_{\beta}^{\mathscr{F}}(N, r, \lambda)\right)$. In the special case $\lambda_{m}=m$, for all $m \in \mathbb{N}$ we will write $\widehat{S}_{\beta}^{\mathscr{F}}(N, r)$ instead of $\widehat{S}_{\beta}^{\mathscr{F}}(N, r, \lambda)$ and in the special case $r_{k}=1$ for all $k \in \mathbb{N}$ and $N(x)=x$ we will write $\widehat{S}_{\beta}^{\mathscr{F}}(\lambda)$ instead of $\widehat{S}_{\beta}^{\mathscr{F}}(N, r, \lambda)$. If we take $\beta=1$, then we obtain the set of all almost $\lambda_{r}$-statistically convergent sequence $\widehat{S}^{\mathscr{F}}(N, r, \lambda)$. If $r_{k}=1$ for all $k \in \mathbb{N}$ then we will write $\widehat{S}_{\beta}^{\mathscr{F}}(N, \lambda)$ instead of $\widehat{S}_{\beta}^{\mathscr{F}}(N, r, \lambda)$.

Definition 2. Let $\lambda=\left(\lambda_{m}\right)$ be as above, let $N$ be an Orlicz function, let $r=\left(r_{k}\right)$ be any sequence of strictly positive real numbers, and let $0<\beta \leq 1$ be given. We define the following sets:

$$
\begin{aligned}
& \widehat{w}_{\beta}^{\mathscr{F}}(N, r, \lambda) \\
& =\left\{X \in w^{\mathscr{F}}: \lim _{m \rightarrow \infty} \frac{1}{\lambda_{m}^{\beta}} \sum_{k \in I_{m}}\left[N\left(\frac{d\left(t_{k n}(X), X_{0}\right)}{s}\right)\right]^{r_{k}}=0,\right. \\
& \text { uniformly in } n, s>0\}, \\
& \widehat{w}_{\beta}^{\mathscr{F}}(N, r, \lambda)_{0} \quad \\
& =\left\{X \in w^{\mathscr{F}}: \lim _{m \rightarrow \infty} \frac{1}{\lambda_{m}^{\beta}} \sum_{k \in I_{m}}\left[N\left(\frac{d\left(t_{k n}(X), \overline{0}\right)}{s}\right)\right]^{r_{k}}=0,\right. \\
& \text { uniformly in } n, s>0\} .
\end{aligned}
$$

$$
\begin{gathered}
\widehat{w}_{\beta}^{\mathscr{F}}(N, r, \lambda)_{\infty} \\
=\left\{X \in w^{\mathscr{F}}: \sup _{m, n} \frac{1}{\lambda_{m}^{\beta}} \sum_{k \in I_{m}}\left[N\left(\frac{d\left(t_{k n}(X), \overline{0}\right)}{s}\right)\right]^{r_{k}}<\infty,\right. \\
s>0\} .
\end{gathered}
$$

If $X \in \widehat{w}_{\beta}^{\mathscr{F}}(N, r, \lambda)$, we say that $X$ is strongly almost $\lambda_{r^{-}}$ convergent of order $\beta$ with respect to the Orlicz function $N$. In this case we write $X_{k} \rightarrow X_{0}\left(\widehat{w}_{\beta}^{\mathscr{F}}(N, r, \lambda)\right)$.

(i) If we take $r_{k}=1$ for all $k \in \mathbb{N}$, then we get $\widehat{w}_{\beta}^{\mathscr{F}}(N, r, \lambda)=\widehat{w}_{\beta}^{\mathscr{F}}(N, \lambda), \widehat{w}_{\beta}^{\mathscr{F}}(N, r, \lambda)_{0}=\widehat{w}_{\beta}^{\mathscr{F}}(N, \lambda)_{0}$, and $\widehat{w}_{\beta}^{\mathscr{F}}(N, r, \lambda)_{\infty}=\widehat{w}_{\beta}^{\mathscr{F}}(N, \lambda)_{\infty}$.

(ii) If we take $N(x)=x$ and $r_{k}=1$ for all $k \in \mathbb{N}$, then we get $\widehat{w}_{\beta}^{\mathscr{F}}(N, r, \lambda)=\widehat{w}_{\beta}^{\mathscr{F}}(\lambda), \widehat{w}_{\beta}^{\mathscr{F}}(N, r, \lambda)_{0}=\widehat{w}_{\beta}^{\mathscr{F}}(\lambda)_{0}$, and $\widehat{w}_{\beta}^{\mathscr{F}}(N, r, \lambda)_{\infty}=\widehat{w}_{\beta}^{\mathscr{F}}(\lambda)_{\infty}$.

(iii) If we take $\beta=1$ and $r_{k}=1$ for all $k \in \mathbb{N}$, then we get $\widehat{w}_{\beta}^{\mathscr{F}}(N, r, \lambda)=\widehat{w}^{\mathscr{F}}(N, \lambda), \widehat{w}_{\beta}^{\mathscr{F}}(N, r, \lambda)_{0}=\widehat{w}^{\mathscr{F}}(N, \lambda)_{0}$, and $\widehat{w}_{\beta}^{\mathscr{F}}(N, r, \lambda)_{\infty}=\widehat{w}^{\mathscr{F}}(N, \lambda)_{\infty}$.

\section{Main Results}

In this section we give the main results of this paper.

The proof of each of the following results is straightforward, so we state the following.

Theorem 3. Let $r=\left(r_{k}\right)$ be bounded, let $\lambda=\left(\lambda_{m}\right)$ be as above, and let $0<\beta \leq 1$; then $\widehat{w}_{\beta}^{\mathscr{F}}(N, r, \lambda)_{0} \subset \widehat{w}_{\beta}^{\mathscr{F}}(N, r, \lambda) \subset$ $\widehat{w}_{\beta}^{\mathscr{F}}(N, r, \lambda)_{\infty}$.

Theorem 4. Let $r=\left(r_{k}\right)$ be bounded, let $\lambda=\left(\lambda_{m}\right)$ be as above, and let $0<\beta \leq 1$; then $\widehat{w}_{\beta}^{\mathscr{F}}(N, r, \lambda)$, $\widehat{w}_{\beta}^{\mathscr{F}}(N, r, \lambda)_{0}$, $\widehat{w}_{\beta}^{\mathscr{F}}(N, r, \lambda)_{\infty}$, and $\widehat{S}_{\beta}^{\mathscr{F}}(N, r, \lambda)$ are closed under the operations of addition and scalar multiplication.

Theorem 5. Let $r=\left(r_{k}\right)$ be bounded, let $\lambda=\left(\lambda_{m}\right)$ be as above, let $0<\beta \leq 1$, and let $N_{1}, N_{2}$ be Orlicz functions; then

(i) $\widehat{w}_{\beta}^{\mathscr{F}}\left(N_{1}, r, \lambda\right)_{0} \cap \widehat{w}_{\beta}^{\mathscr{F}}\left(N_{2}, r, \lambda\right)_{0} \subset \widehat{w}_{\beta}^{\mathscr{F}}\left(N_{1}+N_{2}, r, \lambda\right)_{0}$,

(ii) $\widehat{w}_{\beta}^{\mathscr{F}}\left(N_{1}, r, \lambda\right) \cap \widehat{w}_{\beta}^{\mathscr{F}}\left(N_{2}, r, \lambda\right) \subset \widehat{w}_{\beta}^{\mathscr{F}}\left(N_{1}+N_{2}, r, \lambda\right)$,

(iii) $\widehat{w}_{\beta}^{\mathscr{F}}\left(N_{1}, r, \lambda\right)_{\infty} \cap \widehat{w}_{\beta}^{\mathscr{F}}\left(N_{2}, r, \lambda\right)_{\infty} \subset \widehat{w}_{\beta}^{\mathscr{F}}\left(N_{1}+N_{2}, r\right.$, $\lambda)_{\infty}$.

Theorem 6. Let $0<r_{k} \leq q_{k}$ and $\left(q_{k} / r_{k}\right)$ be bounded, let $\lambda=$ $\left(\lambda_{m}\right)$ be as above, and let $0<\beta \leq 1$; then $\widehat{w}_{\beta}^{\mathscr{F}}(N, q, \lambda) \subseteq$ $\widehat{w}_{\beta}^{\mathscr{F}}(N, r, \lambda)$.

Theorem 7. Let $r=\left(r_{k}\right)$ be bounded, let $\lambda=\left(\lambda_{m}\right)$ be as above, and let $0<\beta \leq 1$. If $\lim _{\inf _{m \rightarrow \infty}}\left(\lambda_{m}^{\beta} / m^{\beta}\right)>0$, then $\widehat{S}_{\beta}^{\mathscr{F}}(N, p) \subset \widehat{S}_{\beta}^{\mathscr{F}}(N, r, \lambda)$. 
Proof. For given $\varepsilon>0$ we have

$$
\begin{aligned}
& \left\{k \leq m:\left[N\left(\frac{d\left(t_{k n}(X), X_{0}\right)}{s}\right)\right]^{r_{k}} \geq \varepsilon\right\} \\
& \quad \supset\left\{k \in I_{m}:\left[N\left(\frac{d\left(t_{k n}(X), X_{0}\right)}{s}\right)\right]^{r_{k}} \geq \varepsilon\right\}
\end{aligned}
$$

and so

$$
\begin{aligned}
& \frac{1}{m^{\beta}}\left|\left\{k \leq m:\left[N\left(\frac{d\left(t_{k n}(X), X_{0}\right)}{s}\right)\right]^{r_{k}} \geq \varepsilon\right\}\right| \\
& \geq \frac{\lambda_{m}^{\beta}}{m^{\beta}} \frac{1}{\lambda_{m}^{\beta}}\left|\left\{k \in I_{m}:\left[N\left(\frac{d\left(t_{k n}(X), X_{0}\right)}{s}\right)\right]^{r_{k}} \geq \varepsilon\right\}\right| .
\end{aligned}
$$

Hence we get $x \in \widehat{S}_{\beta}^{\mathscr{F}}(N, r, \lambda)$.

Theorem 8. Let $r=\left(r_{k}\right)$ be bounded, let $\lambda=\left(\lambda_{m}\right)$ be as above, and let $0<\beta \leq \gamma \leq 1$; then $\widehat{S}_{\beta}^{\mathscr{F}}(N, r, \lambda) \subseteq \widehat{S}_{\gamma}^{\mathscr{F}}(N, r, \lambda)$.

Proof. The following inequality yields the proof:

$$
\begin{aligned}
& \frac{1}{\lambda_{m}^{\gamma}}\left|\left\{k \in I_{m}:\left[N\left(\frac{d\left(t_{k n}(X), X_{0}\right)}{s}\right)\right]^{r_{k}} \geq \varepsilon\right\}\right| \\
& \leq \frac{1}{\lambda_{m}^{\beta}}\left|\left\{k \in I_{m}:\left[N\left(\frac{d\left(t_{k n}(X), X_{0}\right)}{s}\right)\right]^{r_{k}} \geq \varepsilon\right\}\right| .
\end{aligned}
$$

Theorem 9. Let $r=\left(r_{k}\right)$ be bounded, let $\lambda=\left(\lambda_{m}\right)$ be as above, let $0<\beta \leq \gamma \leq 1$, and let $N$ be an Orlicz function; then

(i) $\widehat{w}_{\beta}^{\mathscr{F}}(N, r, \lambda) \subseteq \widehat{w}_{\gamma}^{\mathscr{F}}(N, r, \lambda)$,

(ii) $\widehat{w}_{\beta}^{\mathscr{F}}(N, r, \lambda)_{0} \subseteq \widehat{w}_{\gamma}^{\mathscr{F}}(N, r, \lambda)_{0}$,

(iii) $\widehat{w}_{\beta}^{\mathscr{F}}(N, r, \lambda)_{\infty} \subseteq \widehat{w}_{\gamma}^{\mathscr{F}}(N, r, \lambda)_{\infty}$.

Proof. It is easy so it is omitted.

Theorem 10. If $\lim r_{k}>0$ and $X$ is strongly almost $\lambda_{r^{-}}$ convergent of order $\beta$ to $X_{0}$, with respect to the Orlicz function $N$, then $X_{0}$ is unique.

Proof. Suppose that $X_{k} \rightarrow X_{0}\left(\widehat{w}_{\beta}^{\mathscr{F}}(N, r, \lambda)\right), X_{k} \rightarrow$ $Y_{0}\left(\widehat{w}_{\beta}^{\mathscr{F}}(N, r, \lambda)\right)$, and $\lim r_{k}=\ell>0$; then there exist $s_{1}$ and $s_{2}$ such that

$$
\lim _{m} \frac{1}{\lambda_{m}^{\beta}} \sum_{k \in I_{m}}\left[N\left(\frac{d\left(t_{k n}(X), X_{0}\right)}{s_{1}}\right)\right]^{r_{k}}=0,
$$

$\lim _{m} \frac{1}{\lambda_{m}^{\beta}} \sum_{k \in I_{m}}\left[N\left(\frac{d\left(t_{k n}(X), Y_{0}\right)}{s_{2}}\right)\right]^{p_{k}}=0, \quad$ uniformly in $n$.
Let $s=\max \left(2 s_{1}, 2 s_{2}\right)$. Then we have

$$
\begin{aligned}
& \lim _{m} \frac{1}{\lambda_{m}^{\beta}} \sum_{k \in I_{m}}\left[N\left(\frac{d\left(X_{0}, Y_{0}\right)}{s}\right)\right]^{r_{k}} \\
& \leq \frac{G}{\lambda_{m}^{\beta}} \sum_{k \in I_{m}}\left[N\left(\frac{d\left(t_{k n}(X), X_{0}\right)}{s_{1}}\right)\right]^{r_{k}} \\
&+\frac{G}{\lambda_{m}^{\beta}} \sum_{k \in I_{m}}\left[N\left(\frac{d\left(t_{k n}(X), Y_{0}\right)}{s_{2}}\right)\right]^{r_{k}} \rightarrow 0,
\end{aligned}
$$

where $\sup _{k} r_{k}=H$ and $G=\max \left(1,2^{H-1}\right)$. Thus

$$
\lim _{m} \frac{1}{\lambda_{m}^{\beta}} \sum_{k \in I_{m}}\left[N\left(\frac{d\left(X_{0}, Y_{0}\right)}{s}\right)\right]^{r_{k}}=0 .
$$

Also, since clearly

$$
\lim _{k}\left[N\left(\frac{d\left(X_{0}, Y_{0}\right)}{s}\right)\right]^{r_{k}}=\left[N\left(\frac{d\left(X_{0}, Y_{0}\right)}{s}\right)\right]^{\ell},
$$

therefore $\left[N\left(d\left(X_{0}, Y_{0}\right) / s\right)\right]^{\ell}=0$. Hence $X_{0}=Y_{0}$.

Theorem 11. Let $\lambda=\left(\lambda_{m}\right)$ be as above; then $\widehat{w}^{\mathscr{F}}(N, \lambda)_{\infty}=$ $\widehat{m}^{\mathscr{F}}(N)_{\infty}$ and $\widehat{w}^{\mathscr{F}}(\lambda)_{\infty}=\ell_{\infty}^{\mathscr{F}}$, where

$$
\begin{gathered}
\widehat{m}^{\mathscr{F}}(N)_{\infty}=\left\{X \in w^{\mathscr{F}}: \sup _{k, n}\left[N\left(\frac{d\left(t_{k n}(X), \overline{0}\right)}{s}\right)\right]<\infty,\right. \\
\text { for some } s>0\} .
\end{gathered}
$$

Proof. Let $X \in \widehat{w}^{\mathscr{F}}(N, \lambda)_{\infty}$. Then we can find a constant $K_{1}>$ 0 such that

$$
\begin{aligned}
& \frac{1}{\lambda_{1}}\left[N\left(\frac{d\left(t_{k n}(X), \overline{0}\right)}{s}\right)\right] \\
& \quad \leq \frac{1}{\lambda_{m}} \sum_{k \in I_{m}}\left[N\left(\frac{d\left(t_{k n}(X), \overline{0}\right)}{s}\right)\right] \\
& \quad \leq K_{1} \text { for each } k, n .
\end{aligned}
$$

Hence $X \in \widehat{m}^{\mathscr{F}}(N)_{\infty}$.

Conversely, if $X \in \widehat{m}^{\mathscr{F}}(N)_{\infty}$ we can find a constant $K_{2}>$ 0 such that $N\left(d\left(t_{k n}(X), \overline{0}\right) / s\right) \leq K_{2}$ for each $k, n$ and so

$$
\frac{1}{\lambda_{m}} \sum_{k \in I_{m}} N\left(\frac{d\left(t_{k n}(X), \overline{0}\right)}{s}\right) \leq \frac{K_{2}}{\lambda_{m}} \sum_{k \in I_{m}} 1 \leq K_{2} \quad \text { for each } k, n .
$$


Thus $X \in \widehat{w}^{\mathscr{F}}(N, \lambda)_{\infty}$. Similar proof can be made for the other.

In Theorems 12 and 13, we will assume that the sequence $r=\left(r_{k}\right)$ is bounded and $0<h=\inf _{k} r_{k} \leq r_{k} \leq \sup _{k} p_{k}=H<$ $\infty$. From now on, $\sum_{1}$ will be denoting the sum over $k \in I_{m}$ with $d\left(t_{k n}(X), X_{0}\right) \geq \varepsilon$ and $\sum_{2}$ will be denoting the sum over $k \in I_{m}$ with $d\left(t_{k n}(X), X_{0}\right)<\varepsilon$.

Theorem 12. Let $\lambda=\left(\lambda_{m}\right)$ be as above and let $\beta, \gamma \in(0,1]$ such that $0<\beta \leq \gamma \leq 1$; then $\widehat{w}_{\beta}^{\mathscr{F}}(N, r, \lambda) \subset \widehat{S}_{\gamma}^{\mathscr{F}}(\lambda)$.

Proof. One has $X \in \widehat{w}_{\beta}^{\mathscr{F}}(N, r, \lambda)$; then we have

$$
\begin{aligned}
& \frac{1}{\lambda_{m}^{\beta}} \sum_{k \in I_{m}}\left[N\left(\frac{d\left(t_{k n}(X), X_{0}\right)}{s}\right)\right]^{r_{k}} \\
& \geq \frac{1}{\lambda_{m}^{\gamma}} \sum_{1}\left[N\left(\frac{d\left(t_{k n}(X), X_{0}\right)}{s}\right)\right]^{r_{k}} \\
& \geq \frac{1}{\lambda_{m}^{\gamma}} \sum_{1} \min \left([N(\sigma)]^{h},[N(\sigma)]^{H}\right), \quad \sigma=\frac{\varepsilon}{s} \\
& \geq \frac{1}{\lambda_{m}^{\gamma}}\left|\left\{k \in I_{m}: d\left(t_{k n}(X), X_{0}\right) \geq \varepsilon\right\}\right| \\
& \cdot \min \left([N(\sigma)]^{h},[N(\sigma)]^{H}\right) .
\end{aligned}
$$

Hence $X \in \widehat{S}_{\gamma}^{\mathscr{F}}(\lambda)$.

Theorem 13. Let $\lambda=\left(\lambda_{m}\right)$ be as above and let $\beta, \gamma \in(0,1]$ such that $0<\beta \leq \gamma \leq 1, X \in \ell_{\infty}^{\mathscr{F}}$, and $\lim _{m \rightarrow \infty}\left(\lambda_{m} / \lambda_{m}^{\beta}\right)=1$; then $\widehat{S}_{\beta}^{\mathscr{F}}(\lambda) \subset \widehat{w}_{\gamma}^{\mathscr{F}}(N, r, \lambda)$.

Proof. Suppose that $X \in \ell_{\infty}^{\mathscr{F}}$ and $X_{k} \rightarrow X_{0}\left(\widehat{S}_{\gamma}^{\mathscr{F}}(\lambda)\right)$. Since $X$ is bounded, there exists a constant $B>0$ such that $d\left(t_{k n}(X), X_{0}\right) \leq B$. Let $\varepsilon>0$, and then we have

$$
\begin{aligned}
\frac{1}{\lambda_{m}^{\gamma}} \sum_{k \in I_{m}}\left[N\left(\frac{d\left(t_{k n}(X), X_{0}\right)}{s}\right)\right]^{r_{k}} & \\
\leq & \frac{1}{\lambda_{m}^{\beta}} \sum_{1}\left[N\left(\frac{d\left(t_{k n}(X), X_{0}\right)}{s}\right)\right]^{r_{k}} \\
& +\frac{1}{\lambda_{m}^{\beta}} \sum_{2}\left[N\left(\frac{d\left(t_{k n}(X), X_{0}\right)}{s}\right)\right]^{r_{k}} \\
\leq & \frac{1}{\lambda_{m}^{\beta}} \sum_{1} \max \left\{\left[N\left(\frac{B}{s}\right)\right]^{h},\left[N\left(\frac{B}{s}\right)\right]^{H}\right\} \\
& +\frac{1}{\lambda_{m}^{\beta}} \sum_{2}\left[N\left(\frac{\varepsilon}{s}\right)\right]^{r_{k}}
\end{aligned}
$$

$$
\begin{aligned}
\leq & \max \left\{[N(C)]^{h},[N(C)]^{H}\right\} \\
& \cdot \frac{1}{\lambda_{n}^{\beta}}\left|\left\{k \in I_{m}: d\left(t_{k n}(X), X_{0}\right) \geq \varepsilon\right\}\right| \\
& +\frac{\lambda_{m}}{\lambda_{m}^{\beta}} \max \left\{[N(\delta)]^{h},[N(\delta)]^{H}\right\}, \quad \frac{B}{s}=C, \frac{\varepsilon}{s}=\delta .
\end{aligned}
$$

Hence $X \in \widehat{w}_{\gamma}^{\mathscr{F}}(N, r, \lambda)$.

Let $\lambda=\left(\lambda_{m}\right)$ and $\mu=\left(\mu_{m}\right)$ be two sequences such that $I_{m} \subset J_{m}\left(\lambda_{m} \leq \mu_{m}\right)$ for all $m \in \mathbb{N}$, where $I_{m}=\left[m-\lambda_{m}+1, m\right]$ and $J_{m}=\left[m-\mu_{m}+1, m\right]$. Suppose also that the parameters $\beta$ and $\gamma$ are fixed real numbers such that $0<\beta \leq \gamma \leq 1$. We will now give the following inclusion relations.

Theorem 14. Let $\lambda=\left(\lambda_{m}\right)$ and $\mu=\left(\mu_{m}\right)$ be defined as above, let $N$ be an Orlicz function, and let $\beta, \gamma \in(0,1]$ such that $0<$ $\beta \leq \gamma \leq 1$. Then the following assertions hold true.

(i) If

$$
\liminf _{m \rightarrow \infty} \frac{\lambda_{m}^{\beta}}{\mu_{m}^{\gamma}}>0
$$

$$
\text { then } \widehat{S}_{\gamma}^{\mathscr{F}}(N, \mu) \subseteq \widehat{S}_{\beta}^{\mathscr{F}}(N, \lambda) .
$$

(ii) If

$$
\lim _{n \rightarrow \infty} \frac{\mu_{m}}{\lambda_{m}^{\gamma}}=1
$$

$$
\text { then } \widehat{S}_{\beta}^{\mathscr{F}}(N, \lambda) \subseteq \widehat{S}_{\gamma}^{\mathscr{F}}(N, \mu) \text {. }
$$

Proof. (i) It is easy so it is omitted.

(ii) Suppose that $X \in \widehat{S}_{\beta}^{\mathscr{F}}(N, \lambda)$ and (17) is satisfied. Since $I_{m} \subset J_{m}$, we may write

$$
\begin{aligned}
& \frac{1}{\mu_{m}^{\gamma}}\left|\left\{k \in J_{m}:\left[N\left(\frac{d\left(t_{k n}(X), X_{0}\right)}{s}\right)\right] \geq \varepsilon\right\}\right| \\
& =\frac{1}{\mu_{m}^{\gamma}} \mid\left\{m-\mu_{m}+1<k\right. \\
& \left.\quad \leq m-\lambda_{m}:\left[N\left(\frac{d\left(t_{k n}(X), X_{0}\right)}{s}\right)\right] \geq \varepsilon\right\} \mid \\
& \leq \\
& \quad\left(\frac{1}{\mu_{m}^{\gamma}}\left|\left\{k \in I_{m}:\left[N\left(\frac{d\left(t_{k n}(X), X_{0}\right)}{s}\right)\right] \geq \varepsilon\right\}\right|\right. \\
& \quad+\frac{1}{\lambda_{m}^{\beta}}\left|\left\{k \in I_{m}^{\gamma}:\left[N\left(\frac{d\left(t_{k n}(X), X_{0}\right)}{s}\right)\right] \geq \varepsilon\right\}\right| \\
& \leq\left(\frac{\mu_{m}^{\gamma}}{\lambda_{m}^{\gamma}}-1\right) \\
& \quad+\frac{1}{\lambda_{m}^{\beta}}\left|\left\{k \in I_{m}:\left[N\left(\frac{d\left(t_{k n}(X), X_{0}\right)}{s}\right)\right] \geq \varepsilon\right\}\right|
\end{aligned}
$$

for all $m \in \mathbb{N}$. Hence $X \in \widehat{S}_{\gamma}^{\mathscr{F}}(N, \mu)$. 
Theorem 15. Let $\lambda=\left(\lambda_{m}\right)$ and $\mu=\left(\mu_{m}\right)$ be defined as above, let $N$ be an Orlicz function, and let $\beta, \gamma \in(0,1]$ such that $0<$ $\beta \leq \gamma \leq 1$. Then the following assertions hold true.

(i) If (16) holds, then $\widehat{w}_{\gamma}^{\mathscr{F}}(N, \mu) \subset \widehat{w}_{\beta}^{\mathscr{F}}(N, \lambda)$,

(ii) Let condition (17) be satisfied and $X \in \ell_{\infty}^{\mathscr{F}}$; then $\widehat{w}_{\beta}^{\mathscr{F}}(N, \lambda) \subset \widehat{w}_{\gamma}^{\mathscr{F}}(N, \mu)$.

Proof. It is easy so it is omitted.

Theorem 16. Let $\lambda=\left(\lambda_{m}\right)$ and $\mu=\left(\mu_{m}\right)$ be defined as above, let $N$ be an Orlicz function, and let $\beta, \gamma \in(0,1]$ such that $0<$ $\beta \leq \gamma \leq 1$. Then

(i) if (16) holds, then $\widehat{w}_{\gamma}^{\mathscr{F}}(N, \mu) \subset \widehat{S}_{\beta}^{\mathscr{F}}(N, \lambda)$;

(ii) let condition (17) be satisfied and $X \in \ell_{\infty}^{\mathscr{F}}$; then $\widehat{S}_{\beta}^{\mathscr{F}}(N, \lambda) \subset \widehat{w}_{\gamma}^{\mathscr{F}}(N, \mu)$.

Proof. (i) It is easy so it is omitted.

(ii) Suppose that $X \in \widehat{S}_{\beta}^{\mathscr{F}}(N, \lambda)$ and let $X=\left(X_{k}\right)$ be bounded; then there exists a constant $B>0$ such that $d\left(t_{k n}(X), X_{0}\right) \leq B$. Then we may write

$$
\begin{aligned}
& \frac{1}{\mu_{m}^{\gamma}} \sum_{k \in I_{m}}\left[N\left(\frac{d\left(t_{k n}(X), X_{0}\right)}{s}\right)\right] \\
&=\frac{1}{\mu_{m}^{\gamma}} \sum_{k \in J_{m}-I_{m}}\left[N\left(\frac{d\left(t_{k n}(X), X_{0}\right)}{s}\right)\right] \\
&+\frac{1}{\mu_{m}^{\gamma}} \sum_{k \in I_{m}}\left[N\left(\frac{d\left(t_{k n}(X), X_{0}\right)}{s}\right)\right] \\
& \leq\left(\frac{\mu_{m}-\lambda_{m}^{\gamma}}{\mu_{m}^{\gamma}}\right) B+\frac{1}{\mu_{m}^{\gamma}} \sum_{k \in I_{m}}\left[N\left(\frac{d\left(t_{k n}(X), X_{0}\right)}{s}\right)\right] \\
&=\left(\frac{\mu_{m}}{\mu_{m}^{\gamma}}-1\right) B+\frac{1}{\mu_{m}^{\gamma}} \sum_{1}\left[N\left(\frac{d\left(t_{k n}(X), X_{0}\right)}{s}\right)\right] \\
&+\frac{1}{\mu_{m}^{\gamma}} \sum_{2}\left[N\left(\frac{d\left(t_{k n}(X), X_{0}\right)}{s}\right)\right] \\
& \leq\left(\frac{\mu_{m}}{\lambda_{m}^{\beta}}-1\right) B \\
&+\frac{B}{\lambda_{m}^{\beta}} \mid\left\{k \in I_{m}:\left[\frac{\mu_{m}}{\lambda_{m}^{\gamma} \varepsilon}\right]\right.
\end{aligned}
$$

for all $n \in \mathbb{N}$. Hence we obtain $X \in \widehat{w}_{\gamma}^{\mathscr{F}}(N, \mu)$.

\section{Conflict of Interests}

The authors declare that there is no conflict of interests regarding the publication of this paper.

\section{References}

[1] L. A. Zadeh, "Fuzzy sets," Information and Computation, vol. 8, pp. 338-353, 1965.

[2] M. Matloka, "Sequences of fuzzy numbers," Busefal, vol. 28, pp. 28-37, 1986.

[3] H. Altınok, Y. Altın, and M. Ișık, "Statistical convergence and strong $\mathrm{p}$-Cesàro summability of order $\beta$ in sequences of fuzzy numbers," Iranian Journal of Fuzzy Systems, vol. 9, no. 2, pp. 6373, 2012.

[4] H. Altınok, "On $\lambda$-statistical convergence of order $\beta$ of sequences of fuzzy numbers," International Journal of Uncertainty, Fuzziness and Knowledge-Based Systems, vol. 20, no. 2, pp. 303-314, 2012.

[5] S. Aytar and S. Pehlivan, "Statistically monotonic and statistically bounded sequences of fuzzy numbers," Information Sciences, vol. 176, no. 6, pp. 734-744, 2006.

[6] R. Çolak, H. Altınok, and M. Et, "Generalized difference sequences of fuzzy numbers," Chaos, Solitons and Fractals, vol. 40, no. 3, pp. 1106-1117, 2009.

[7] M. Et, H. Altınok, and R. Çolak, "On $\lambda$-statistical convergence of difference sequences of fuzzy numbers," Information Sciences, vol. 176, no. 15, pp. 2268-2278, 2006.

[8] A. Gökhan, M. Et, and M. Mursaleen, "Almost lacunary statistical and strongly almost lacunary convergence of sequences of fuzzy numbers," Mathematical and Computer Modelling, vol. 49, no. 3-4, pp. 548-555, 2009.

[9] F. Nuray, "Lacunary statistical convergence of sequences of fuzzy numbers," Fuzzy Sets and Systems, vol. 99, no. 3, pp. 353$355,1998$.

[10] Ö. Talo and F. Başar, "On the space $b v_{p}(F)$ of sequences of $p$ bounded variation of fuzzy numbers," Acta Mathematica Sinica, English Series, vol. 24, no. 7, pp. 1205-1212, 2008.

[11] H. Fast, "Sur la convergence statistique," Colloquium Mathematicum, vol. 2, pp. 241-244, 1951.

[12] A. Alotaibi, M. Mursaleen, and S. A. Mohiuddine, "Statistical approximation for periodic functions of two variables," Journal of Function Spaces and Applications, vol. 2013, Article ID 491768, 5 pages, 2013.

[13] J. S. Connor, "The statistical and strong p-Cesaro convergence of sequences," Analysis, vol. 8, pp. 47-63, 1988.

[14] M. Et, "Strongly almost summable difference sequences of order m defined by a modulus," Studia Scientiarum Mathematicarum Hungarica, vol. 40, no. 4, pp. 463-476, 2003.

[15] M. Et, M. Çınar, and M. Karakaş, "On $\lambda$-statistical convergence of order $\alpha$ of sequences of function," Journal of Inequalities and Applications, vol. 2013, article 204, 2013.

[16] J. A. Fridy, "On statistical convergence," Analysis, vol. 5, no. 4, pp. 301-313, 1985.

[17] M. Iş1k, "Strongly almost $(w, \lambda, q)$-summable sequences," Mathematica Slovaca, vol. 61, no. 5, pp. 779-788, 2011.

[18] S. A. Mohiuddine, A. Alotaibi, and M. Mursaleen, "Statistical convergence of double sequences in locally solid riesz spaces," Abstract and Applied Analysis, vol. 2012, Article ID 719729, 9 pages, 2012.

[19] M. Mursaleen and S. A. Mohiuddine, "Korovkin type approximation theorem for almost and statistical convergence," in Nonlinear Analysis: Stability, Approximation, and Inequalities, vol. 68 of Springer Optimization and Its Applications, pp. 487494, Springer, New York, NY, USA, 2012. 
[20] B. C. Tripathy, "Matrix transformation between some classes of sequences," Journal of Mathematical Analysis and Applications, vol. 206, no. 2, pp. 448-450, 1997.

[21] A. D. Gadjiev and C. Orhan, "Some approximation theorems via statistical convergence," Rocky Mountain Journal of Mathematics, vol. 32, no. 1, pp. 129-138, 2002.

[22] R. Çolak, "Statistical convergence of order $\alpha$ " in Modern Methods in Analysis and Its Applications, pp. 121-129, Anamaya, New Delhi, India, 2010.

[23] R. Çolak and Ç. A. Bektaş, “ $\lambda$-statistical convergence of order a," Acta Mathematica Scientia, Series B: English Edition, vol. 31, no. 3, pp. 953-959, 2011.

[24] M. Başarır, S. Altundăg, and M. Kayıkçı, "On some generalized sequence spaces of fuzzy numbers defined by a sequence of Orlicz functions," Rendiconti del Circolo Matematico di Palermo, vol. 59, no. 2, pp. 277-287, 2010.

[25] G. G. Lorentz, "A contribution to the theory of divergent sequences," Acta Mathematica, vol. 80, pp. 167-190, 1948.

[26] I. J. Maddox, "A new type of convergence," Mathematical Proceedings of the Cambridge Philosophical Society, vol. 83, no. 1, pp. 61-64, 1978. 


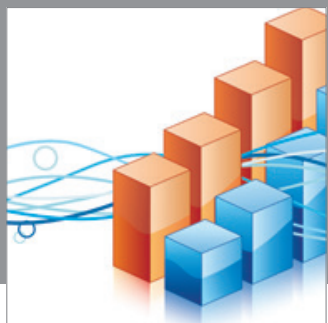

Advances in

Operations Research

mansans

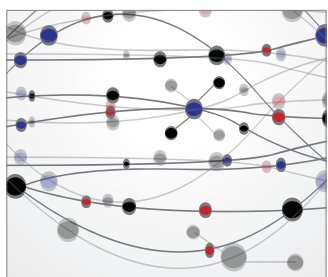

The Scientific World Journal
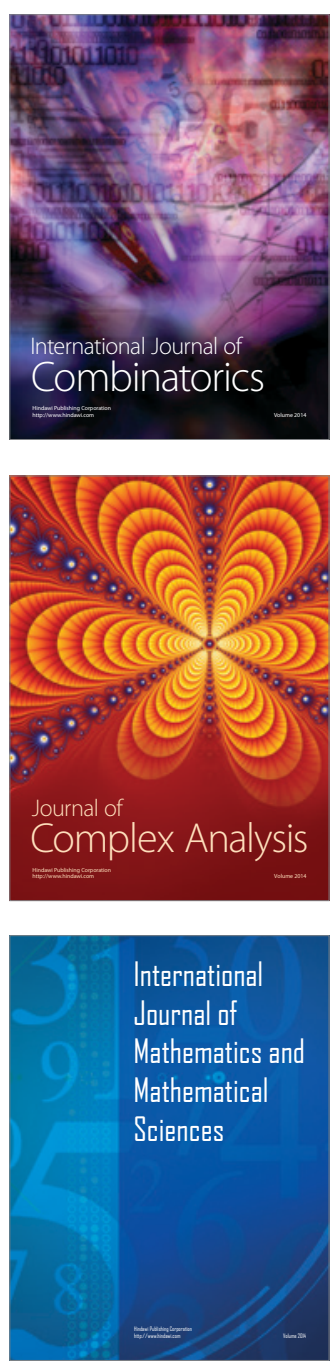
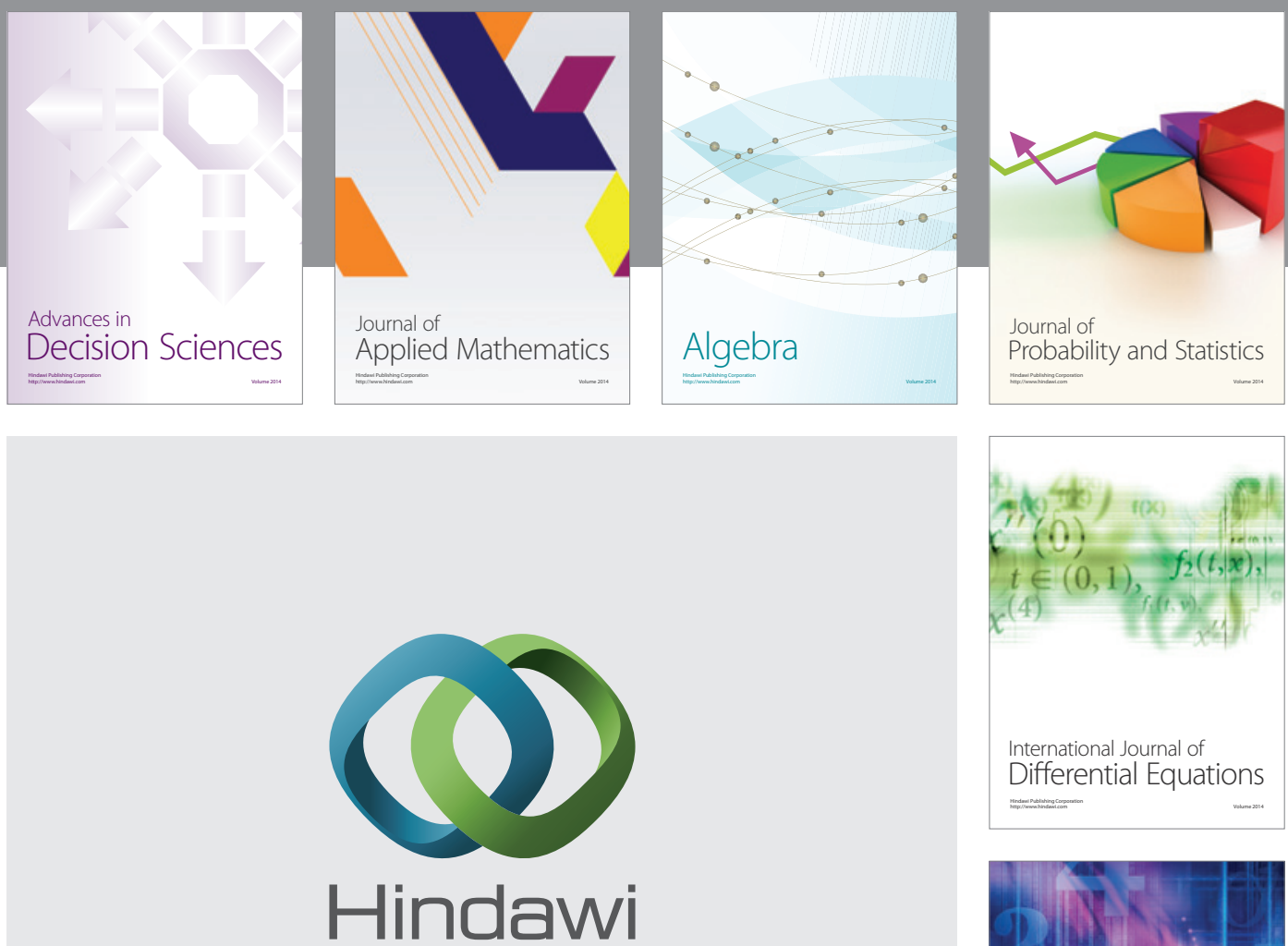

Submit your manuscripts at http://www.hindawi.com
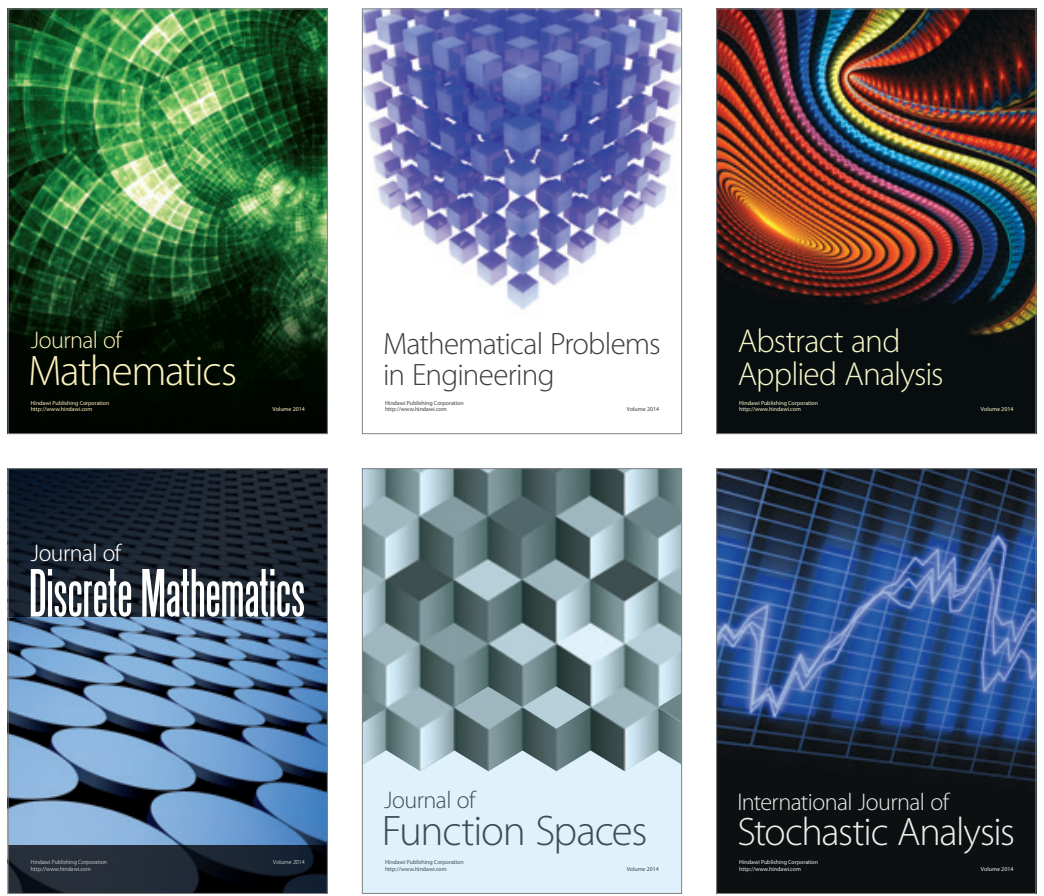

Journal of

Function Spaces

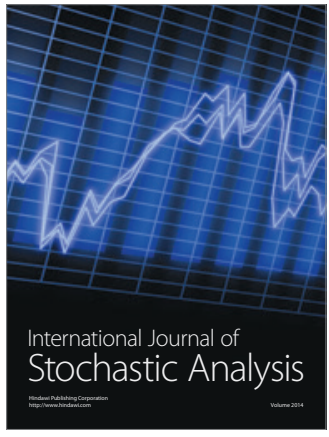

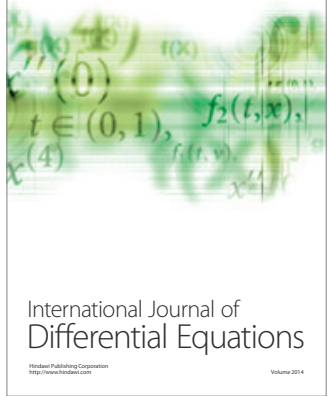
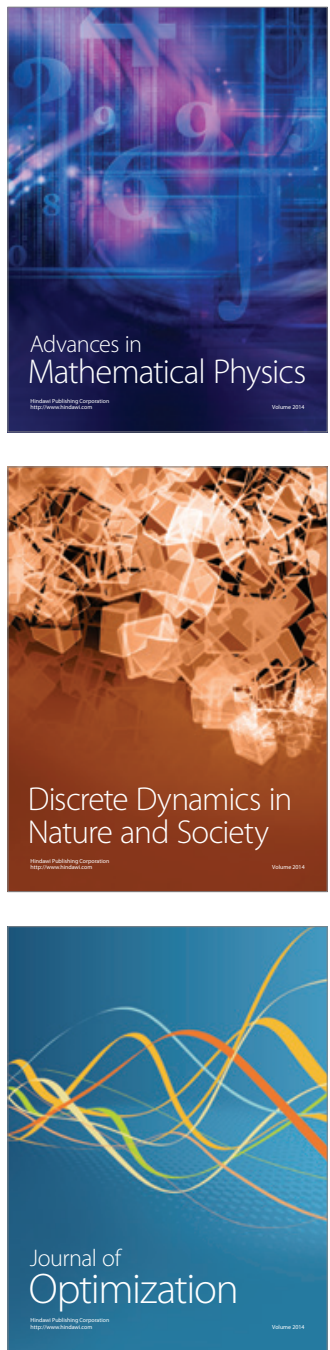